\title{
El liderazgo pedagógico y la implicación del profesorado como factores de éxito en centros de entornos desfavorecidos en España
}

\author{
Educational leadership and teacher involvement as success factors in schools in disadvantaged \\ areas of Spain
}

\author{
Pàmies-Rovira, Jordi ${ }^{(1)}$; Senent-Sánchez, Joan María ${ }^{(2)}$; Essomba Gelabert, Miquel Angel ${ }^{(1)}$
}

(1) Universidad Autónoma de Barcelona (2) Universidad de Valencia

\begin{abstract}
Resumen
Las investigaciones en educación y desigualdades muestran que el entorno socioeconómico de las familias es uno de los factores que condicionan en mayor medida el logro de los resultados académicos. Sin embargo, sin negar la influencia de los factores externos, las investigaciones ponen también de relieve el papel clave que pueden tener la institución educativa y los agentes escolares en las trayectorias y el logro del éxito académico de los jóvenes en situación de mayor vulnerabilidad. Y constatan que escuelas con similares perfiles de estudiantes alcanzan logros diferenciados

Este artículo presenta los resultados de un proyecto de investigación cuyo objetivo ha sido identificar los factores que contribuyen al éxito académico en escuelas de educación primaria y secundaria obligatoria situadas en entornos socioeconómicos desfavorecidos de cuatro grandes ciudades en España (Barcelona, Madrid, Sevilla y Valencia). La investigación muestra la importancia que sobre los resultados académicos tienen las dimensiones institucionales internas y la incidencia del liderazgo pedagógico de la dirección y del profesorado en el logro académico.
\end{abstract}

Fecha de recepción 10 Mayo 2016

Fecha de aprobación 22 Noviembre 2016

Fecha de publicación 25 Noviembre 2016

\section{Palabras clave:}

entornos desfavorecidos, éxito académico, liderazgo pedagógico, profesorado

\begin{abstract}
Research into education and inequalities shows that families' socio-economic background is one of the factors that has a determinig effect on the achievement of academic results. However, while not denying the influence of external factors, research also highlight the key role the school and school agents can play in more vulnerable young people's educational pathways and academic achievements. It also finds that schools with similar student profiles display different levels of achievement.

This article presents the results of a research project aimed at identifying factors that contribute to academic success in primary and secondary schools located in disadvantaged socio-economic areas in four major cities in Spain (Barcelona, Madrid, Seville and Valencia). This research shows the importance of internal institutional dimensions for academic results and the impact of the educational leadership displayed by school directors and teachers on academic achievement
\end{abstract}

\section{Keywords:}

disadvantaged backgrounds, academic achievement, educational leadership, teachers

\section{Reception Date}

2016 May 10

Approval Date

2016-November-22

Publication Date:

2016-November-25
Las investigaciones en educación y desigualdades muestran que el entorno socioeconómico de las familias es uno de los factores que condicionan en mayor medida el logro de los resultados académicos. Una constatación que cuestiona el alcance real de la función de la escuela y pone en evidencia que estas cumplen de forma escasa el objetivo de reducir las desigualdades (Ferrer, 2009). Sin embargo, diversos informes e investigaciones 
(PISA, PIRLS, Duru-Bellat \& Suchaut, 2005, entre otros) muestran también los factores del sistema educativo y de centro que inciden sobre los resultados del alumnado y ponen de manifiesto que, en algunos sistemas educativos, las desigualdades inciden en menor medida en la experiencia educativa y los resultados académicos de los ciudadanos. Estos datos conviven con los que constatan que escuelas con similares perfiles de estudiantes alcanzan logros diferenciados (Dupriez \& Dumay, 2006; Murillo, 2007; Sammons, Hillman \& Mortimore, 1995) y con trabajos que confirman que la acción de la escuela es clave para reducir las desigualdades pero también que la mejora de la equidad refuerza los niveles de excelencia del sistema educativo (Sammons, 2007; Suchaut, 2005).

Barber y Mourshed (2007) al estudiar las características de 25 sistemas educativos que participaron en el programa PISA para conocer las causas del éxito educativo concluyen que los factores fundamentales de este éxito son tres: a) la calidad del profesorado, b) la mejora de los procesos de enseñanza-aprendizaje en el aula y c) la consecución de la mejora del nivel para todos los estudiantes. Los autores señalan cómo la calidad del sistema tiene como techo la calidad de los docentes pero constatan también el efecto que tiene la mejora de los procesos de enseñanza y aprendizaje en el aula y el efecto que ejerce el liderazgo escolar en la mejora de los resultados. Bolivar (2010) señala que el efecto de este liderazgo que en general resulta ser un "efecto-director", es un factor indirecto que contribuye a construir las condiciones para que el profesorado trabaje bien en las aulas. Por su parte, Hargreaves (2010) señala que los directores que promueven la mejora de la escuela pueden lograr mejoras en el logro de los estudiantes, es decir, que las acciones que impulsan los directores impactan tanto sobre los profesores, como sobre los estudiantes.

En el presente artículo se presentan datos empíricos acerca del liderazgo pedagógico y las funciones del profesorado. La presentación se realiza a partir de los resultados de un proyecto de investigación que ha tenido como objetivo principal identificar los factores que contribuyen al éxito académico y a la continuidad educativa de los jóvenes en centros educativos de educación primaria $\mathrm{y}$ secundaria obligatoria situados en entornos socioeconómicos desfavorecidos de 4 grandes ciudades en España (Barcelona, Madrid, Sevilla y Valencia). Al abordar el efecto escuela, la investigación muestra la importancia que sobre los resultados académicos tienen las dimensiones institucionales internas y los procesos educativos y en este caso la importancia que tiene el liderazgo pedagógico de la dirección y del profesorado.

\section{El liderazgo pedagógico: motor de cambio}

Trabajos como los de Marzano, Waters \& McNulty (2005) muestran como los equipos directivos pueden marcar una importante diferencia en la educación del alumnado y en la calidad de las propias escuelas. Unos datos que corroboran el Informe McKinsey (Barber $\&$ Mourshed, 2007) y la propia OCDE (Pont, Nusche \& Moorman, 2008) al concluir que la mejora de una institución escolar depende en gran medida de la labor de equipos directivos que ejerzan un liderazgo eficaz, capaz de dinamizar el centro e impulsar la mejora continua. Estas investigaciones sitúan el liderazgo como el segundo factor interno en la escuela que más relevancia tiene en el alcance de los logros de aprendizaje, tras la que sería la acción directa que ejerce en este logro el profesorado. El informe TALIS (2013) resalta también la importancia que tiene el liderazgo escolar para favorecer la eficacia del trabajo del profesorado en la escuela. Sin embargo, en este informe España obtiene una de las puntuaciones más bajas en liderazgo pedagógico y en liderazgo administrativo.

Por su parte, en el informe APA (2010) llevado a cabo en Colorado, se identifican también los factores de mejora de ocho escuelas con una elevada proporción de estudiantes de familias de bajos ingresos. Entre estos factores el informe señala la existencia de altas expectativas para todos los 
estudiantes, el apoyo individualizado para el alumnado con dificultades, la participación activa de los docentes en la toma de decisiones y el desarrollo de una cultura de colaboración entre ellos. Pero también otros factores notables, como la flexibilidad de la escuela para utilizar los recursos, la estabilidad y en especial la consistencia del liderazgo. Un amplio número de investigaciones muestra el papel trascendental del liderazgo escolar en el logro de la eficacia y mejora de las escuelas (Beauchamp y Parsons, 2012; Pont, Nusche \& Moorman, 2009). Trabajos como los de Scheerens y Bosker (1997), Teddlie y Reynolds (2000), Harris y Mujis (2002) o Townsend (2007) dejan constancia de ello, y también de cómo el liderazgo resulta ser una influencia indirecta que aparece mediada por otros factores imbricados tanto en la estructura y organización escolar, como en la práctica docente y el ambiente de la escuela y de aula. Sin que sea posible contar con evidencias que permitan apuntar cuál es el estilo de liderazgo más eficaz - aunque el liderazgo distribuido y participativo parecen serlo (Harris \& Muijs, 2002) - y que como señalan Pont, Nusche y Moorman (2008) no existan estudios a gran escala que proporcionen un vínculo directo entre el liderazgo, el aprendizaje estudiantil y los resultados de escuela, existen estudios de caso que informan que el líder escolar ejerce una importante función. Este, cuya labor resulta en especial eficaz cuando se centra en aspectos relacionados con la enseñanza y el aprendizaje (Harris y Chapman, 2002), crea o no las condiciones necesarias para influir en las motivaciones y condiciones de trabajo de los docentes, los que son responsables en última instancia de moldear las prácticas escolares y el aprendizaje eficaz.

La función directiva y su liderazgo educativo es pues, fundamental para la formulación de una visión del centro compartida por el profesorado y por toda la comunidad educativa, y para la creación de un plan estratégico anual con unos objetivos medibles, definidos alrededor de las necesidades académicas, lingüísticas y de desarrollo del alumnado (García \& del Campo, 2012).

\section{La implicación del profesorado: clave para el éxito}

El profesorado es concebido como agente clave para apuntalar el éxito del alumnado y al ser el principal gestor de los procesos de enseñanza-aprendizaje y el artífice de la organización del centro, su competencia profesional, su rol docente y su actitud personal resultan determinantes para el éxito. Así la calidad del profesorado resulta para muchas investigaciones (Aaronson, Barrow \& Sander 2007; Kane \& Staiger 2008; Muijs \& Reynolds, 2010; Rivkin, Hanushek \& Kain 2005; Rockoff 2004, entre otras) ser un factor esencial para explicar la efectividad de las escuelas y la diferencia de resultados que obtienen. En este sentido, resulta relevante contemplar aportaciones como la de la Ofsted (2009), que ofrece una mirada para la mejora que debería inspirar también la formación docente y la supervisión educativa. Para la Ofsted (2009) la contribución del profesorado al éxito escolar viene marcada por la interacción que este genera con el alumnado y con otros miembros de la comunidad educativa. Con relación a las interacciones que lleva a cabo con el primero, destaca tres principios: que el profesorado conozca y profundice en las necesidades educativas de todos sus alumnos; que focalice su atención en las causas y no en los efectos de una potencial desafección escolar; y que lleve a cabo una evaluación del alumnado que vaya más allá de ser una medición del rendimiento académico a través de pruebas objetivas.

Las investigaciones aportan resultados coincidentes acerca del perfil de un profesor eficaz (Attali \& Bressoux, 2002; Lessard, 2006, entre otros). Se trata de un profesor facilitador que desarrolla las actividades de manera estructurada, trabaja introduciendo dificultades de forma progresiva a sus estudiantes, a quienes plantea preguntas, ofrece evaluaciones y retroalimentación positiva. $Y$ es estricto. Para otros investigadores (Meirieu \& Le Dû 2006; 
Perrenoud, 1999, entre otros), el profesor eficaz, es un profesor que organiza y gestiona las situaciones de aprendizaje. Este profesor coloca a sus estudiantes, a través de la acción, en una situación de investigación y solicita a los alumnos que resuelvan sus problemas. Es un defensor convencido del principio de "educabilidad". Cree que todos los alumnos tienen potencial para aprender; defiende una escuela que prepara para la vida; y apuesta por la realización de experiencias de aprendizaje basadas en la acción, normalmente en colaboración con otros profesores en el marco de la comprensividad; propone problemas y retos a sus alumnos. Crea un clima de buena comunicación y confianza en el aula, de manera que todos los alumnos se sientan seguros y confiados; observa y escucha al alumnado con atención, y se interroga a sí mismo con respecto a su labor. Más allá de las lecciones de clases, este profesor está siempre dispuesto a crear un ambiente estimulante cognitivamente, a movilizar a los alumnos para realizar actividades, a crear un entorno rico en propuestas de aprendizaje y es consciente de que su rol educativo no se restringe a la clase. Su actuación global como educador en todo momento favorece que sus alumnos sientan el conjunto de la escuela como un lugar amable. Siempre está dispuesto a proponer desafíos de aprendizaje (Meirieu \& Le Dû, 2006)

\section{Método}

Los resultados de este artículo pertenecen al proyecto financiado por el Ministerio de Economía y Competitividad $\mathrm{I}+\mathrm{D}+\mathrm{I}$ titulado "Éxito y desigualdad educativa en centros escolares de entornos desfavorecidos". El estudio ha abordado diversos niveles de análisis y combinado metodologías cuantitativas y cualitativas, entre los que se encuentran el análisis de políticas educativas a nivel internacional, la explotación de bases de datos cuantitativas (como PIRLS y PISA) y una fase cualitativa en la que se han realizado 24 estudios de casos en centros escolares de cinco entornos urbanos españoles: Barcelona
(5) y su área metropolitana (5), Sevilla (4), Madrid (5) y Valencia (5).

En la selección de los centros educativos se ha contemplado que fuesen centros de educación primaria y de educación secundaria obligatoria, públicos y concertados, que se encontraran en barrios de condiciones socioeconómicas desfavorecidas y que tuvieran resultados educativos por encima de las escuelas de su ciudad situadas en entornos similares. Para la selección de los centros se ha contado con el apoyo y la colaboración de las administraciones escolares de cada Comunidad Autónoma $^{[1]}$. Se ha seleccionado en cada ciudad 5 escuelas $^{[2]}: 4$ centros públicos, 2 de educación primaria y 2 de educación secundaria, y 1 centro concertado. En cada uno de los centros (24) se han realizado entrevistas en profundidad a directores (1), inspectores (1) $\mathrm{y}$ técnicos municipales de educación (1) y organizado grupos de discusión con estudiantes (1), profesorado (1) y familias (1). En total se han realizado 55 entrevistas - se habían previsto un total de 60 pero 2 inspectores declinaron participar en la investigación y en 3 casos el técnico municipal estaba involucrado en más de una escuela -, y 75 grupos de discusión.

Se han obtenido los permisos de los diferentes agentes para la realización del trabajo de campo. Por un lado el de las administraciones, por otro el de los centros y sus claustros de profesores, las familias y el alumnado que han participado tanto en las entrevistas como en los grupos de discusión. Se ha pactado la manera de devolución de los resultados en forma de informe particular para cada uno de los centros, que, como es habitual, han sido anonimizados.

\section{Resultados}

El contexto de la investigación: los centros educativos

Los centros que han participado en la investigación están situados en zonas densamente pobladas, barrios de clase trabajadora y lugar de llegada de migraciones interiores en los años 60 y de migrantes 
internacionales en las últimas décadas. Estos barrios con tasas de paro elevadas, son considerados conflictivos por la mayor parte de los entrevistados, en especial por los jóvenes. Así se expresaban algunos de ellos, al preguntarles, que les gustaba más del barrio:

- Es que en el barrio no hay nada que nos guste

- Lo que está bien del barrio es que hay metro y puedes salir”. (Alumnado, $\mathrm{AMB} / \mathrm{C} 5)$

La mayoría de los estudiantes de los centros provienen de familias de clase trabajadora y nivel socioeconómico bajo $\mathrm{y}$ entre un $30-50 \%$ son hijos e hijas de familias inmigradas. De forma general, después de haber sido centros poco deseados en el mercado educativo de la ciudad, mantienen en la actualidad unas cifras consolidadas de matriculación, un aspecto que se considera positivo desde los propios centros y resultado del trabajo realizado en los últimos años. Sin embargo, esto no significa que la mayor parte de estos centros no puedan seguir siendo construidos en el barrio desde la conflictividad. Sirva esta frase, recogida en uno de los grupos de discusión con familias y que ejemplifica la realidad expuesta: " $a$ mí me dijeron que habían matado a una niña en el lavabo" (Grupo de familias B/3). En contraposición incluso aquellas familias que transmiten una imagen negativa del centro inscriben en él a sus hijos e hijas, cuando estos requieren de procesos de acompañamiento escolar mas individualizados.

El liderazgo pedagógico como motor de cambio

El liderazgo es el primer elemento de la cultura institucional al que se refieren los agentes entrevistados. Este liderazgo recae de forma general en las direcciones de los centros. El reconocimiento explícito e implícito del equipo directivo como líderes del proyecto, se hace evidente en el siguiente extracto:

"Es muy buen comunicador [el director] y transmite mucho, y también es capaz de argumentar de manera que tú ves que aquello está pasando. [...] $Y$ eso es muy importante. $Y$ este empuje y estas ganas que tienen el equipo directivo, esto lo transmiten, tanto en el claustro de docentes, como a los chicos, como a los padres. $Y$ creo que es una cadena que se contagia. $Y$ esto es imprescindible". (Familias, centro $\mathrm{AMB} / \mathrm{C} 3$ )

En los centros de la investigación se detectan dos situaciones en cuanto a la eficacia de este liderazgo. Por un lado, aquellos casos en que lo es por su extensa trayectoria (de más de 10 años con este reconocimiento) y aquellos en que siendo reciente supone una ruptura y novedad, es decir, que se percibe el "antes y el después", como un cambio que ha sido muy favorable para la evolución de los centros:

"El director ha cambiado mucho (el centro), porqué mi hermano cuando venía era muy conflictivo esto y entonces él veía las cosas porque era profesor. Entonces cuando se pasó a ser director, empezó a cambiar todo, porque aquí había muchas bandas antes y mi madre estaba preocupada por qué viniera aquí, por las bandas. Y claro llegué aquí y no había ningún problema de bandas y de nada porqué todo lo había cambiado él” (Alumnado, AMB/C 3)

Tampoco existe homogeneidad en el tipo de liderazgo ejercido, y en consecuencia se han detectado centros con un liderazgo distributivo y altamente valorado por este hecho, y otros con unas características más personalistas y jerárquicas en su ejercicio:

"Esto es como el arca de Noé. Hay dos elefantes, dos tigres, dos leones, dos moscas, dos mosquitos, dos iguanas, dos no sé qué. Pero a todo el mundo le he dado un remo. Y uno rema así, el otro asá, uno hace una cosa, el otro hace otra... Pero a todos les he dicho hacia dónde tenemos que ir". (Director, centro B/C3)

En el caso de los liderazgos distributivos se acompaña de un conocimiento de los puntos 
fuertes y débiles del equipo de profesores. Esta situación permite poder potenciarlos o solucionarlos, a la vez que impulsar una colaboración intensa de todos los agentes educativos, como expone el equipo directivo de uno de los centros,

"A nivel de organigrama nosotros trabajamos mucho, muy conjuntamente con los coordinadores de ciclo porque ellos son los que conocen la realidad de cada ciclo, la realidad de cada aula, sabes, y entonces todas las decisiones se planean conjuntamente con ellos” (Equipo directivo, B/C1).

En los casos de liderazgos distributivos el profesorado considera que el director tienen la capacidad de acompañar, de seducir y de respetar, situación que les lleva a algunos a considerar que:

- "El equipo directivo tiene la habilidad de saber invitar a todos a colaborar.

- "No es lo mismo obligar que invitar, y X (el director) invita"

- "X (el director) es un poco como Dios: está a todas partes y lo sabe todo" (Profesorado, AMB/C3)

En el caso de los liderazgos jerárquicos y/o personalistas las decisiones recaen más en una sola figura, por lo que esta persona deviene la concentración de atributos, características y valores que son valorados por el resto, es decir que se presenta como un ejemplo a seguir:

"Otra clave del éxito es el ejemplo. El ejemplo.[...]. Tienes que dar un cierto ejemplo de autoridad sin... Es decir, saber que estás allí, no hacerte invisible, no encerrarte en un despacho, salir, mirar, estar, escuchar...". (Director, centro B/C3)

En ambos casos se desprende también que la implicación del equipo directivo representa un ejemplo de trabajo para el resto del profesorado. Desde la dirección se transmite "una motivación que se pega" y representa un modelo de implicación y trabajo para el resto del profesorado.
Ahora bien, ¿en qué dimensiones hemos constatado que ha incidido de forma preferente el liderazgo de las direcciones, facilitando el desarrollo de una mejor respuesta educativa? Pasemos a considerar algunas:

- En la aceptación en positivo de la imagen pública del centro a partir del alumnado que escolariza. Las direcciones han impulsado un proyecto de centro que no persigue modificar la composición social del alumnado y de sus familias. Acepta de forma positiva los jóvenes que escolariza y a partir del análisis de sus necesidades impulsa las respuestas educativas. Asimismo la mayor parte del profesorado procura no reproducir el discurso sobre la presencia de alumnado minoritario, inmigrante $\mathrm{o}$ de necesidades educativas especiales (n.e.e.) como una situación anómala a resolver. Así lo expresaba un responsable municipal:

"Son centros en general inclusivos, que es otra clave del éxito. $Y$ de no valorar el prestigio del centro en función de la tipología de alumnado que tengas, sino en función del tipo de trabajo que realices en el centro" (Representante municipal, $\mathrm{AMB} / \mathrm{C} 5$ )

- En el análisis constante y la búsqueda de respuestas y soluciones a los retos cotidianos. El compromiso del equipo directivo y del profesorado en la mejora del centro y los resultados educativos de sus alumnos se traduce en un análisis constante sobre el centro, así como una reflexión continuada sobre las acciones que se llevan a cabo. El uso de la evaluación -tanto interna como externa- para la mejora y la búsqueda de respuestas ante los problemas identificados son ejemplos de cómo los centros se plantean sus acciones para el cambio y de que no se trabaja desde la improvisación. Podemos afirmar que en los centros imperan las propuestas posibilistas y en positivo, un indicador claro que refleja la presencia en el centro de la cultura de la transformación. 
- En la elaboración de un proyecto de centro fuerte y compartido que asegura la posibilidad de adquirir las competencias a todo el alumnado. La dirección ha impulsado un proyecto en esta línea y junto a ello el profesorado del centro educativo goza de libertad en sus iniciativas. El equilibrio entre libertad y confianza depositada en los docentes por parte del equipo directivo genera un alto nivel de compromiso y de vinculación al centro.

- En el impulso a la participación en programas y proyectos educativos y el establecimiento de redes de apoyo con el fin de adecuar y enriquecer la respuesta educativa del centro. Desde el liderazgo de la dirección se ha promocionado la apertura de las escuelas al barrio y el trabajo en red con las entidades, en un programa preestablecido que se revisa cada curso. Desde estos centros se considera esencial una apertura hacia el entorno y trabajar en colaboración con las entidades y servicios del barrio y de la ciudad.

- En la comunicación, coordinación y colaboración con los centros educativos del entorno. De igual modo que señalábamos antes el establecimiento de redes de apoyo, en la investigación hemos detectado como la colaboración, comunicación y coordinación con otros centros educativos del entornos tanto de primaria como de secundaria era mucho mayor de la habitual y se realizaba más allá de que fueran centros de la misma red (públicos o concertados) traduciendo una clara preocupación educativa por el barrio.

- En el establecimiento de estructuras que permitan, desde la colaboración con otras instancias, contribuir a dar respuesta adecuada a las necesidades de todos los alumnos, desde una perspectiva inclusiva. La colaboración supone que en el centro coexistan los órganos de gestión normativos con las estructuras creadas ex proceso y que pretenden dar una respuesta a las desigualdades educativas desde una perspectiva inclusiva y desde la flexibilidad organizativa. Sirvan de ejemplos las "nuevas estructuras" que algunos centros han creado: como el equipo dinamizador, formado por el equipo directivo y un grupo de profesores, o la Comisión social, formada por agentes internos y externos al centro y ejemplo de trabajo en red. O la Asociación de Amigos del Instituto, que por iniciativa del equipo de dirección se ha constituido como nueva entidad y que ofrece becas a los estudiantes.

- En el desarrollo de un clima y una cultura escolar positiva. En los centros se ha podido constatar la existencia de una cultura escolar positiva que viene favorecida por la creación desde la dirección de espacios que favorecen el dialogo, la reflexión y la participación entre el profesorado. Podríamos afirmar que en todos los centros ocupan un lugar importante las relaciones humanas. Una situación que impulsa el desarrollo el establecimiento de vínculos afectivos entre los miembros de la comunidad educativa y contribuye al desarrollo de un clima y una cultura escolar positiva.

- En la flexibilidad en la interpretación de las normas de la administración educativa y su defensa ante instancias superiores. En muchos casos las peculiaridades del centro no permiten que las dinámicas de estos se ajusten a las directrices normativas. Algunos directores nos comentaban que "vivir al filo de la ley (o de la norma administrativa) comenzaba a ser una necesidad, así como tener que defenderlo y explicarlo ante la inspección educativa $\mathrm{u}$ otras instancias de la administración educativa.

- En el desarrollo de estrategias de formación del profesorado ad-hoc sostenidas en el tiempo. La mayor parte de los centros cuentan, impulsadas por la dirección, con estrategias de formación dirigidas a los docentes. Estas se centran en el trabajo cooperativo, la formación de competencias básicas, las herramientas digitales, la formación de mediadores, la 
coeducación o la comprensión lectora. En este sentido, los docentes destacan la influencia que ha realizado la formación sobre la programación didáctica. Sin embargo, se reconoce que no todos los profesores se involucran en estos procesos.

El profesorado: implicación en el proyecto de centro

Relacionado en cierta medida con el liderazgo, emergen los procesos de toma de decisiones por parte del equipo de docentes. Tanto el profesorado como el director de la mayor parte de los centros de la investigación señalan que la forma más habitual de toma de decisiones es el consenso, en los diferentes órganos de representación donde tienen presencia: claustros, equipos docentes, comisiones, etc. De forma independiente al tipo de liderazgo, el director delega su confianza en el resto de profesorado, hecho que comporta fortalecer el sentimiento de responsabilidad compartida en el proyecto común e incrementa la fidelización y el compromiso de los docentes. Existe un sentimiento de contagio de la implicación y la voluntad de colaboración y mejora. Las familias tienen esta percepción, y resaltan la implicación de los docentes, en el sentido de que el compromiso del profesorado va más allá de lo que es estrictamente académico.

"Nosotros estamos haciendo de dos grupos, tres. [...] Yo hago trabajar al profesorado 120 horas más de las que le corresponde.[...] El equipo directivo trabaja más horas, y por lo tanto, si nosotros trabajamos más horas en el aula, trabajamos menos horas en la gestión, digamos que tenemos menos horas de reducción, ¿de acuerdo? Los directores de departamento solo tienen una hora de reducción, pero la mayoría de ellos son tutores, por lo tanto ya están haciendo de directores de departamento y de tutores. La mayoría de coordinadores de actividades extraescolares, de informática, coordinador lingüístico, etc., no tienen ninguna hora de reducción.[...] Todo el mundo está trabajando el máximo de horas posibles para que se pueda hacer esto. Se hizo un pacto, en el claustro". (Director, B/C4)

Las familias resaltan también que el compromiso del profesorado va más allá de lo que es estrictamente académico y que dedican horas más allá del horario escolar:

"Yo veo que es gente realmente implicada, y gente que se preocupa por la educación integral del alumno. No sólo por enseñarle sociales o matemáticas". (Familias, B/C3)

"Sí, la implicación. Lo comentábamos antes, por el concierto de Navidad, o por la obra de teatro... Están trabajando fuera de horas y no tienen ningún problema. Y si los niños van por la tarde, porqué tienen que ensayar la obra, pues los profesores también están allí. [...] Hay un feeling distinto de ir a la escuela y después ir para casa" (Familias, B/C4)

La importancia de este factor es, según las direcciones, crucial, pues genera unas dinámicas que favorecen los buenos resultados, mediante el trabajo conjunto y la existencia de objetivos compartidos:

"Yo también pienso que es un proyecto muy integrado por la mayor parte de los integrantes del claustro. Es decir, uno de nuestros grandes activos es que tenemos un profesorado en general muy comprometido, que se hace muy suyo este proyecto de escuela y hace que dedique más esfuerzo que la media de los docentes. Yo creo que en esta línea" (Profesorado, AMB/C5)

Además, se identifica que esta doble causalidad está alimentada por la confianza que el equipo directivo, desde un primer momento, ha depositado en todos los docentes del centro:

"Yo he dado mucha confianza a todo el mundo. En un principio no regalé casi nada, pero lo primero que hice fue sacar la hoja de firmas. Aquí, se entraba y se firmaba, y se ponía la hora en que habías firmado. Todo el profesorado, ¿eh? Pues yo lo primero que hice fue sacar esto. Porque yo confiaba en que todo el mundo tiene 
ganas de venir pronto.[...] Claro, confiar en ellos de que no hacía falta que firmasen, ha llevado a que cada vez haya más responsabilidad a la hora de faltar". (Director, centro B/C4)

Asimismo, en muchos casos la implicación está acompañada de un sentimiento de vinculación del profesorado con el centro y con el proyecto colectivo:

"Todo el mundo ha ido entrando en una dinámica en que todo el mundo tiene una tarea, todo el mundo tiene una tarea en este centro, a parte de la típica docente.[...] Cosas muy sencillas, pero todo el mundo se siente vinculado a la vida, a algo del Instituto, a algo que nos agarra al Instituto". (Director, centro M/C3)

En realidad algunos de los profesores ponen de manifiesto que un motivo por el cual todos los profesores se encuentran tan implicados con el centro es que la mayoría de ellos reside en el distrito donde se encuentra el colegio.

"Creo que eso hace también, no a lo mejor conscientemente, implicarte más, pero sí que de alguna forma ves que la realidad de los niños es tu realidad al final" (Profesor, M/C4)

Esta vinculación y compromiso es perceptible a través de los discursos del alumnado y de las familias en relación a las expectativas hacia los estudiantes y el trato que reciben. También respecto a la elevada sensibilidad por parte del profesorado y del equipo directivo de las condiciones de vida cotidianas de los estudiantes. Ilustra esta situación, por ejemplo, que ante la inexistencia de comedor en un centro de secundaria, se tramiten becas de comedor, para que los estudiantes de las familias más necesitadas puedan acudir al comedor del centro de primaria adyacente:

"hay en este momento cinco o seis alumnos que se están quedando en el comedor de primaria. Nosotros como equipo directivo pues tenemos el compromiso de estar cada día de la semana uno de nosotros para dar apoyo (en el comedor)" (Directora, B/C4)

En casi todos los casos los estudiantes y las familias ponen en relieve que el profesorado y el equipo directivo están disponibles para comentar lo que sea necesario y que existe un conocimiento "real" de los niños y jóvenes, como se pone en relieve en esta frase de un niño de primaria y el siguiente reconocimiento de las familias:

"es que ellos [los profesores] creen más en mí que yo mismo; yo pensaba que no lo podría hacer y mira, pues sí,..." (Estudiante de primaria, $\mathrm{B} / \mathrm{C} 1$ )

"Aquí se cuida, y es verdad que se cuidan. Se cuidan de los chicos y de las familias. $Y$ eso pienso que es uno de los temas para el éxito escolar de nuestro centro". (Familias, centro B/C3)

Los estudiantes hacen una valoración positiva del profesorado. Identifican en él diversas cualidades de carácter general y en sus prácticas docentes en el aula. En el caso de la etapa de educación secundaria, consideran que un buen profesor es aquel que explica bien, no es repetitivo, ofrece un trato de igual a igual, escucha, sabe juzgar, permite y reconoce la capacidad de mejora, es agradable $y$ simpático, da confianza, se muestra estricto en las relaciones, es tolerante y fomenta el trabajo grupal. Algunos de sus profesores consideran que comparten buena parte de estas cualidades:

"Que se sepa, o sea, que te explique bien, o sea que explique a su manera pero cuando tengas una queja que la escuche (...)

"Pues que explique bien, que sepa juzgar y pues no sé, que sea agradable con los compañeros, con los alumnos “

"A mí me gustan mucho los profesores que tratan de igual a igual, que no te traten con inferioridad, como si fuéramos más pequeños! (Alumnos AMB/C3)

Las familias destacan la importancia de la labor del profesorado, y que en el centro todos 
los profesores conozcan a todos los alumnos. Una situación que el profesorado corrobora, en especial en aquellos centros donde la estabilidad de la plantilla es mayor y en los centros privados concertados:

"porque hay chicos que los conoces desde ... y además conoces sus hermanos, su familia ... Llegas a tener un conocimiento de cada uno que si estás un año y te vas a otro centro no puedes tener" (Profesorado, AMB/C5)

Es decir, el profesorado de los centros comparte la preocupación por los estudiantes y sus aprendizajes, pero además en los centros se promueve una educación más allá de lo académico, que no solo valora los aprendizajes curriculares, sino que también aprecia la formación en valores. Los profesores acompañan a los estudiantes y trasmiten un compromiso individual con cada uno de ellos basado en la exigencia y en el afecto:

"Formamos personas e intentamos que estas personas no salgan sólo con conocimientos, sino que sepan de alguna forma compartir, relacionarse" (Director, $A M B / C 4)$

El acompañamiento se percibe en las diversas estrategias que se impulsan en los centros y en especial en la acción tutorial y la orientación educativa, que se considera en la mayoría de las ocasiones una de las prioridades. En este ámbito la labor del profesorado y del equipo directivo es intensa. Ambos se involucran en el seguimiento de las situaciones y la dirección ofrece el apoyo a los profesores, estudiantes y familias, un aspecto valorado por todos los agentes educativos. Por su parte los estudiantes se sienten apoyados por el profesorado no sólo en los aspectos académicos, también en lo personal. Entre los aspectos que los alumnos consideran que convierten a estas en buenas escuelas destacan la actitud de los profesores hacia ellos:

"La actitud que tienen contigo, porque incluso fuera del horario de clase, si tú tienes algún problema familiar o algo y se lo cuentas te ayudan, eso lo tengo yo muy en cuenta". (Alumnado, $\mathrm{AMB} / \mathrm{C} 3$ )

Resulta esta una situación que llega a sorprender a algunos estudiantes y familias en la etapa de educación secundaria, y que colabora a romper ciertas construcciones acerca de esta etapa educativa:

"El trato que hay en este centro no me lo esperaba e $n$ un centro de secundaria, para nada, te lo esperas en un centro de primaria pequeños como estos " (Familias, $B / C 4)$

Sin embargo, en la etapa de secundaria en algunos casos se ha percibido también que algunos estudiantes prefieren que el apoyo que reciban sea estrictamente académico:

"No es que me gustaría....!sino que paso, paso!....Por mucho que le cuentes tus cosas, sigue siendo un profe, no es ni u amigo, ni un hermano, es un profe y ya está" (Alumnos, AMB/C4)

El buen clima al que hemos hecho referencia, reconocido en los centros y las relaciones estrechas del profesorado con los estudiantes y las familias van acompañados de una relación evidente entre la importancia otorgada al respeto a las normas, la exigencia y el aprendizaje que se consigue :

"Estos profes, los que son exigentes, son los que realmente aprendes, isabes?. Claro que están contigo...que enseñan algo,...y es con los que realmente aprendes algo" (Alumnos B/C4)

\section{Discusión}

Las tendencias internacionales (Day et al, 2009) señalan la necesidad de considerar el liderazgo pedagógico como un liderazgo para el aprendizaje, y vincular este liderazgo al aprendizaje de todo el alumnado. Hemos podido constatar como a través de la dirección de las escuelas y del propio profesorado se realizan estos procesos de vinculación y como las acciones del liderazgo se dirigen a la mejora de los logros de todos los estudiantes. 
En estos centros, el sistema relacional se caracteriza por la existencia de un clima positivo junto a un liderazgo fuerte que emerge como uno de los factores clave. En todos los casos este va acompañado de un elevado grado de dedicación del equipo directivo pero también del profesorado en general. De este, alumnado y familias valoran su elevado grado de compromiso académico y personal con cada estudiante, como también habían puesto de relieve otros estudios (Hopkins \& Reynolds, 2002; Joyce et al., 1999; Lein et al., 1997; Leithwood \& Steinbach, 2003; Montgomery et al., 1993; Muijs et al., 2004; Scheerens \& Bosker, 1997).

Sin duda, promover una escuela para el éxito en entornos desfavorecidos exige la implicación de profesionales preparados y comprometidos, que deben actuar a partir de dos grandes ejes. El primero de ellos, contribuir a la clarificación del proyecto de escuela, desde la revisión continuada del contexto escolar. El análisis facilitará conocer la situación del centro y las posibilidades y límites de implementación de las diversas líneas de actuación y estrategias. El segundo, buscar las sinergias y complicidades entre todos los agentes educativos. El trabajo colaborativo y la búsqueda de consensos se impulsa cuando el director delega su confianza en el conjunto del profesorado, una situación que incrementa la fidelización y el compromiso de los docentes con el proyecto de centro. En este artículo hemos intentado aportar evidencias empíricas al respecto $\mathrm{y}$ hemos podido constatar como el rol del liderazgo del director y la implicación del profesorado son un factor de calidad y un medio necesario para luchar contra el fracaso escolar en entornos desaventajados. Sin embargo, los líderes escolares sólo pueden ejercer influencia si cuentan con autonomía y apoyo para tomar decisiones significativas y si sus responsabilidades están bien definidas (OCDE, 2009: 41) una situación poco desarrollada en España, a pesar de las relevantes experiencias existentes (ver Revista de Educación, número extraordinario, 2012).
Al preguntarnos cuáles son las políticas que favorecen una tipología de profesorado más acorde con el rol que en estos contextos socioeducativos se perfila como conducente al éxito, Loeb y colegas (2011) muestran cómo las políticas de gestión y de formación pueden resultar determinantes. En este sentido, las autoras concluyen que las escuelas más eficaces destacan debido, entre otras medidas, a la aplicación de una política caracterizada por la contratación del profesorado con mayor valor añadido, la distribución más equitativa del profesorado novel (asignándolo en menor medida a los grupos desaventajados), la mejora de sus competencias profesionales y la promoción de un liderazgo eficaz y de unas prácticas docentes de calidad. Un aspecto que Johnston y Hayes (2007) consideran clave, al mostrar como el aprendizaje del estudiante está relacionado con el aprendizaje profesional del profesorado. En concreto, constatan que los estudiantes tienden a obtener un mayor éxito si el profesorado participa activamente de las oportunidades de aprendizaje que les ofrece el contexto de su escuela. Este es un aspecto que hemos podido reconocer también en nuestra investigación. Ahora bien, como recoge también Bolivar (2010), estamos en una situación ante la que cabe preguntarse si actuar bajo el principio del liderazgo pedagógico, sin considerar las dimensiones de desigualdad social y educativa será eficaz en sí o, si por el contrario, estamos contribuyendo a reproducir una situación usando planteamientos similares a los que justificamos para crearlos.

\section{Referencias}

Aaronson, D., Barrow, L. \& Sander, W. (2007) Teachers and Student Achievement in the Chicago Public High Schools. Journal of Labor Economics, 25(1). doi: https://doi.org/10.1086/508733

Attali, A. \& Bressoux, P. (2002). L'évaluation des pratiques éducatives dans les premier et second degrés. Rapport pour le Haut Conseil de l'Evaluation de l'Ecole. Recuperado de http://webcom.upmf-grenoble.fr/sciedu/pbressou 
Astolfi, J.P (1992). L'ecole pur aprendre. Paris: ESF.

Barber, M. \& Mourshed, M (2007). How the World's Best-Performing School Systems Come Out on Top. Londres: McKinsey and Company.

Beauchamp, L. \& Parsons, J (2012). Instructional Leadership in Alberta: Research insights from five highly effective schools. Journal of Scholarship \& Practice, 8 (4).

Bolívar, A. (2010). El liderazgo educativo y su papel en la mejora: Una revisión actual de sus posibilidades y limitaciones. Psicoperspectivas, 9(2), 9-33. doi: https://doi.org/10.5027/psicoperspectivas-

Vol9-Issue2-fulltext-112

Day, C., Sammons, P., Hopkins, D., Harris, A., Leithwood, K., Gu, Q., Brown, E., Ahtaridou, E. \& Kington, A. (2009), The Impact of School Leadership on Pupil Outcomes: Final Report, UK Department for Children, Schools and Families Research.

Dupriez, V. \& Dumay, X. (2006). Inequalities in school systems: effect of school structure or of society structure? Comparative Education, 42(2), 243-260. doi: http://doi.org/10.1080/03050060600628074

Duru-Bellat, M. \& Suchaut, B. (2005). Organisation and Context, Efficiency and Equity of Educational Systems: What PISA tells us. European Educational Research Journal, 4(3), 181-194. doi: https://doi.org/10.2304/eerj.2005.4.3.3

Field, S., Kuczera, M., \& Pont, B. (2007). No More Failures: Ten Steps to Equity in Education. Paris: OECD. doi: https://doi.org/10.1787/9789264032606-en

Garcia Alegre, E. \& del Campo Canals, M. (2012). ¿La corresponsabilidad es una estrategia de éxito? Revista de Educación, Número Ext, 220-248. doi: http://doi.org/10.4438/1988-592X-RE-2012EXT-213

Gorard, S. \& Smith, E. (2004). An international comparison of equity in education systems. Comparative Education, 40(1), 15-28. doi: http://doi.org/10.1080/0305006042000184863
Hargreaves, A. (2010). Rethinking school reform: Learning from the inspiring examples of other systems and countries. University of Massachusetts, Lowell Institute. Recuperado de http://associationdatabase.com/aws/

Harris, A., \& Chapman, C. (2002). Leadership in schools facing challenging circumstances. Management in Education, 16(1), 10-13. doi: http://doi.org/10.1177/08920206020160010301

Harris, A., Muijs, D. (2002). Teacher leadership: A review of research. Coventry/London: University of Warwick Institute of Education - General Teaching Council.

Hopkins, D., \& Reynolds, D. (2002). The past, present and future of school improvement. London: DfES.

Johnston, K. \& Hayes, D (2007) Supporting student success at school trought teacher professional learning: The pedagogy of disrupting he default modes of schooling. International Journal of Inclusive Education, 11(3), 371-381. doi: https://doi.org/10.1080/13603110701240666

Joyce, B., Calhoun, E., \& Hopkins, D. (1999). The New Structure of School Improvement Inquiring Schools and Achieving Students. Buckingham: Open University Press.

Kane, T. \& Staiger, D (2008) Estimating Teacher Impacts on Student Achievement: An Experimental Evaluation. NBER Working Paper No. 14607 JEL No. I21. doi: https://doi.org/10.3386/w14607

Leithwood, K., \& Steinbach, R. (2003). Successful leadership for especially challenging schools. In Handbook of educational leadership and management. London: Pearson.

Lessard, C. (2006). Regulação múltipla e autonomia profissional dos professores, comparação entre o Quebec e o Canadá, Educação em Revista, 44. doi: https://doi.org/10.1590/s0102-

46982006000200008

Marzano, R. J., Waters, T. \& McNulty, B. A. (2005). School leadership that works: From research to results. Alexandria, VA: 
Association for Supervision and Curriculum Development.

Montgomery, A., Rossi, R., Legters, N., McDill, E., McPartland, J., \& Stringfield, S. (1993). Educational reforms and students placed at risk: A review of the current state of the art. Washington, DC: US Department of Education, OERI.

Muijs, D., Harris, A., Chapman, C., Stoll, L., \& Russ, J. (2004). Improving Schools in Socioeconomically Disadvantaged Areas - A Review of Research Evidence. School Effectiveness and School Improvement: An International Journal of Research, Policy and Practice, 15(2), 149-175. doi: https://doi.org/10.1076/sesi.15.2.149.30433

Muijs, D., Reynolds, D. (2010). Effective Teaching: Research and Practice. London: Paul Chapman.

Meirieu, Ph. \& Le Dû, M. C (2006). Carta a un joven profesor: por qué enseñar hoy. Barcelona : Graó,

Murillo, J. (2007). School Effectiveness Research in Latin America. In T. Townsend (Ed.), International Handbook of School Effectiveness and Improvement (pp. 75-92). Springer Netherlands. doi: https://doi.org/10.1007/978-1-4020-5747-2_5

OCDE (2007). Understanding the Social Outcomes of Learning. Paris: OECD. doi: https://doi.org/10.1787/9789264068780-en

OCDE (2009). Creating Effective Teaching and Learning Environments: First Results from TALIS.

https://doi.org/10.1787/9789264068780-en

OCDE (2010). PISA 2009 Results Overcoming Social Background: Equity in Learning opportunities and Outcomes. Vol II. Paris: OECD.

OCDE (2011). Against the Odds: Disadvantaged Students Who Succeed in School. OECD Publishing. doi: http://doi.org/10.1787/9789264090873-en

Perrenoud, Ph. (1999). Construir competencias desde la escuela. Santiago de Chile: Dolmen.

Pont, B., D. Nusche., \& Moorman, H. (2008). Improving school leadership. Paris, OCDE.
Recuperado

de

http://www.oecd.org/edu/schoolleadership

Reynolds, D. (2010). Failure Free Education?

The Past, Present and Future of School

Effectiveness and School Improvement.

London: Routledge.

Rivkin, S. G., Hanushek E., \& Kain, J (2005)

Teachers, schools, and academic achievement.

Econometrica 73, no. 2:417-58. doi:

https://doi.org/10.1111/j.1468-

0262.2005.00584.x

Sammons, P. (2007). School Effectiveness and Equity: Making Connections. A review of school effectiveness and improvement makers(pp. 1-77). CfBT.

Sammons, P., Hillman, J., \& Mortimore, P. (1995). Key Characteristics of Effective Schools: A review of school effectiveness research. London: OFSTED.

Scheerens, J. \& Bosker, R. J. (1997). The Foundations of Educational Effectiveness. Oxford: Pergamon.

Suchaut, B. (2005), Un regard sur l'efficacité des dispositifs de lutte contre l'échec scolaire. Regards sur l'actualité, 310, pp. 51-58

TALIS (2013). Estudio internacional sobre la Enseñanza y el Aprendizaje. Informe español. Educa INEE $n^{o}$ 33. Recuperado de http://www.mecd.gob.es/inee/Ultimos_inform es/TALIS-2013.html

Teddie, C. \& Reynolds, D (ed) (2000). The International Handbook of School Effectiveness Research. London, Falmer Press

Teddlie, C., \& Stringfield, S. (1993). Schools Make a Difference: Lessons Learned from a 10-Year Study of School Effects. New York: Teachers College Press.

The Office for Standards in Education (OFSET) (2009) Children's Services and Skills Departmental Report 2008/09. Retrieved from https://www.gov.uk/government/uploads/ system/uploads/attachment data/file/238633 17597.pdf

Townsend, T. (Ed) (2007). International Handbook of School Effectiveness and School Improvement. New York: Springer. doi: https://doi.org/10.1007/978-1-4020-5747-2 


\section{$\underline{\text { Notas }}$}

[1] En algunos casos la participación de la Administración educativa ha sido más proactiva. Agradecemos el apoyo mostrado.

[2] A excepción de Sevilla donde sólo pudieron participar en la investigación 4 centros.

\section{Agradecimientos}

Este artículo ha sido realizado en el marco del proyecto del Ministerio de Economía y Competitividad I+D+I titulado "titulado Éxito y desigualdad educativa en centros escolares de entornos desfavorecidos". EDU2011-23473"

\begin{tabular}{lc}
\hline \multicolumn{1}{c}{ Autores / Authors } & $\begin{array}{c}\text { To know more / } \\
\text { Saber más }\end{array}$ \\
\hline Pàmies Rovira, Jordi (jordi.pamies@uab.cat). & \\
Profesor del Departamento de Pedagogía Sistemática y Social de la Universitat & \\
Autònoma de Barcelona y miembro del grupo de Investigación EMIGRA-CER & ORCID \\
Migraciones. Sus principales líneas de trabajo son "educación, desigualdades y & 0000-0003-1707-4797 \\
migraciones" y "políticas educativas destinadas a la gestión de la diversidad & \\
cultural”. Su dirección postal es Universitat Autònoma de Barcelona. Departamento & \\
Pedagogía Sistemática y Social. Edifici G6-Despacho 145. Campus de Bellaterra. & \\
08193-Bellaterra. Cerdanyola del Vallès (España). & \\
\hline
\end{tabular}

\begin{abstract}
Senent Sánchez, Joan Maria (Joan.M.Senent@uv.es).
Profesor Titular de la Universidad de Valencia en el Dept. de Educación Comparada e $\mathrm{H}^{\mathrm{a}}$ de la Educación. Es Presidente de la Comisión de Programas Internacionales de la Facultad. Sus principales líneas de trabajo son "educación comparada e internacional", "formación del profesorado y de los educadores sociales", "movilidad de estudiantes y docentes", "cooperación al desarrollo en educación" y "dinámica de grupos e innovación educativa en la escuela". Su dirección postal es: Universitat de València, Facultad de Filosofía y Ciencias de la Educación. Dept. Educació Comparada i Hª de l'Educació. Av. Blasco Ibañez, 30. 46010-València (España).
\end{abstract}

Essomba Gelabert, Miquel Angel (miquelangel.essomba@uab.cat).

Profesor del Departamento de Pedagogía Aplicada de la Universidad Autónoma de Barcelona, Director del grupo de investigación ERIC (Equipo de Investigación en Interculturalidad e Inmigración en Cataluña). Sus principales líneas de trabajo son "educación intercultural", "inclusión de minorías" y "metodologías didácticas de inclusión de la diversidad". Su dirección postal es Universitat Autònoma de Barcelona. Departamento Pedagogía Aplicada. Edifici G6-Despacho 257. Campus de Bellaterra. 08193-Bellaterra. Cerdanyola del Vallès (España).

\title{
RELIEVE
}

\section{Revista ELectrónica de Investigación y $\mathbf{E V}$ aluación $\mathbf{E d u c a t i v a ~}$ E-Journal of Educational Research, Assessment and Evaluation}

[ISSN: 1134-4032]

(C) Copyright, RELIEVE. Reproduction and distribution of this articles it is authorized if the content is no modified and their origin is indicated (RELIEVE Journal, volume, number and electronic address of the document).

(C) Copyright, RELIEVE. Se autoriza la reproducción y distribución de este artículo siempre que no se modifique el contenido y se indique su origen (RELIEVE, volumen, número y dirección electrónica del documento). 